Stardust and the Molecules of Life (Why are the Amino Acids Left-Handed?)

R. N. Boyd, T. Kajino, T. Onaka

April 9, 2010

Origin of Matter and Evolution of Galaxies 10 Osaka, Japan

March 8, 2010 through March 10, 2010 
This document was prepared as an account of work sponsored by an agency of the United States government. Neither the United States government nor Lawrence Livermore National Security, LLC, nor any of their employees makes any warranty, expressed or implied, or assumes any legal liability or responsibility for the accuracy, completeness, or usefulness of any information, apparatus, product, or process disclosed, or represents that its use would not infringe privately owned rights. Reference herein to any specific commercial product, process, or service by trade name, trademark, manufacturer, or otherwise does not necessarily constitute or imply its endorsement, recommendation, or favoring by the United States government or Lawrence Livermore National Security, LLC. The views and opinions of authors expressed herein do not necessarily state or reflect those of the United States government or Lawrence Livermore National Security, LLC, and shall not be used for advertising or product endorsement purposes. 


\title{
Stardust and the Molecules of Life \\ (Why are the Amino Acids Left-Handed?)
}

\author{
Richard N. Boyd ${ }^{1,2}$, Toshitaka Kajino ${ }^{2,3}$, and Takashi Onaka ${ }^{3}$ \\ ${ }^{1}$ Lawrence Livermore National Laboratory; \\ ${ }^{2}$ National Astronomical Observatory of Japan; \\ ${ }^{3}$ University of Tokyo, Department of Astronomy, Graduate School of Science
}

Abstract

A mechanism for creating and selecting amino acid chirality is identified, and subsequent chemical replication and galactic mixing that would populate the galaxy with the predominant species will be described. This involves: (1) the spin of the ${ }^{14} \mathrm{~N}$ in the amino acids, or in precursor molecules from which amino acids might be formed, coupling to the chirality of the molecules; 2 ) the neutrinos emitted from the supernova, together with magnetic field from the nascent neutron star or black hole from the supernova selectively destroying one orientation of the ${ }^{14} \mathrm{~N}$, thereby selecting the chirality associated with the other ${ }^{14} \mathrm{~N}$ orientation; (3) amplification by chemical evolution, by which the molecules replicate on a relatively short timescale; and (4) galactic mixing on a longer timescale mixing the selected molecules throughout the galaxy.

\section{Introduction}

A longstanding problem in science has involved the chirality of the amino acids, the molecules from which the proteins on which we depend are formed. Although producing amino acids in the laboratory results in equal populations of left-handed and right-handed molecules (1), those found on Earth are all left-handed (except for one non-chiral amino acid). The cause of this has perplexed scientists for several decades $(2,3)$, and resulted in several explanations of the possible origin of this effect. The existence of mixtures of left- and right-handed amino acids in meteorites, but with a predominance of left-handed ones whenever one was dominant (4-6), strongly suggests that the amino acids are produced in outer space, and that whatever mechanism produces their chirality also operates in outer space.

One mechanism for producing amino acid enantiomerism, i.e., an excess of one chiral state, that seems to be favored by many is that involving the effect of circularly polarized light interacting with the molecules (7). Ultraviolet light has been shown in the laboratory to be capable of introducing molecular chirality (8), and such light is produced from hot stars, albeit as a small fraction of the total (9). However, this mechanism has one basic difficulty, that is, that the circularly polarized light that is produced with one polarization in one place will surely be compensated for in another place by light of the opposite polarization. When averaged over all space this will produce a global enantiomerism of zero. Arguments have been put forth as to how some non-zero enantiomerism could be established, but at least some authors (2) have professed skepticism that they could really work.

Another mechanism involves the weak interaction. Beta-decay from, for example, ${ }^{14} \mathrm{C}$, would produce electrons which, in interacting with the components of the atoms in which they reside, would produce bremsstrahlung, which would be polarized, and would produce a chiral effect (10). However, 
this was estimated to be an extremely small effect, of order one part in $10^{11}(11)$. Although this may be sufficient to tip the balance toward the chirality that would ultimately be established, an enormous amount of subsequent amplification would be essential to produce the $100 \%$ amino acid enantiomerism that is observed on Earth.

We have proposed the Supernova Neutrino Amino Acid Processing, or SNAPP, model (12), which does appear capable of producing a net chirality for the amino acids, and would be expected to produce a considerably larger level of chirality in some instances than other models (12). This model involves (i) the ${ }^{14} \mathrm{~N}$ in the amino acids, or in precursor molecules from which amino acids were formed, coupling to the chirality of the molecules; (ii) the neutrinos emitted from the supernova, together with magnetic field from the nascent neutron star or black hole formed from the supernova, selectively destroying one orientation of the ${ }^{14} \mathrm{~N}$, thereby selecting the chirality associated with the other ${ }^{14} \mathrm{~N}$ orientation; (iii) amplification of the chirality thus selected, both in outer space and once the molecules got to a planet, and (iv) spreading of the chirality established by supernovae throughout the galaxy.

\section{Core Collapse Supernovae}

Stars that begin their lives with masses greater than about eight times the mass of the Sun will ultimately complete all stages of their stellar evolution and end their lives as neutron stars or black holes. In so doing they expel nearly all of their core energy, which results from their gravitational collapse, by emitting $10^{57}$ neutrinos, or $10^{53} \mathrm{ergs}$, in $\sim 10 \mathrm{sec}(13)$. The six flavors of neutrinos and antineutrinos are roughly equal in number (14) and their energies are comparable (although their hierarchy of mean energies is $\left\langle E\left(U_{e}\right)\right\rangle\left\langle\left\langle E\left(\underline{U}_{e}\right)\right\rangle\left\langle\left\langle E\left(U_{x}\right)>=\left\langle E\left(\underline{U}_{x}\right)>, x=\mu\right.\right.\right.\right.$ or $\left.\tau\right)$, with a spectral peak at around $12 \mathrm{MeV}(15)$. The neutrinos have spin of $\hbar / 2$, and the spin of the electron neutrinos, $v_{e}$ (antineutrinos, $\underline{u}_{e}$ ) is anti-aligned (aligned) with their momentum. Another feature of this stellar collapse is that it often generates extremely strong magnetic fields. Figure 1 shows the situation that exists when the supernova emits its $\underline{u}_{\mathrm{S}} \mathrm{s}$ (on which we will focus, as is discussed below); the need for the magnetic field lines to reconnect means that they will be parallel to the $\underline{u}_{\mathrm{e}}$ spin on one side and antiparallel to the $\underline{u}_{\mathrm{e}}$ spin on the other.

\section{Neutrino Interactions and Chiral Selectivity}

What is important to the SNAPP model is that the ${ }^{14} \mathrm{~N}$ that exists in the amino acids will be oriented by the neutron star or black hole magnetic field during the time that the $\underline{u}_{e} s$ are emitted. Thus, on one side, the ${ }^{14} \mathrm{~N}$ spin will be aligned with the $\underline{u}_{e}$ spin, producing a spin projection of $3 \hbar / 2$, and on the other it will be antialigned, giving a spin projection of $\hbar / 2$. In the former case the total angular momentum must be $3 / 2 \hbar$, but it will be a mixture of $3 \hbar / 2$ and $\hbar / 2$ on the other side. The $u_{e} s$ and $\underline{u}_{e} s$ will interact with the ${ }^{14} \mathrm{~N}$ through the charged current weak interaction in the following ways (14):

$$
\begin{aligned}
& \mathrm{U}_{\mathrm{e}}+{ }^{14} \mathrm{~N} \rightarrow{ }^{14} \mathrm{O}+\mathrm{e}^{-}, \mathrm{Q}=-5.14 \mathrm{MeV}, \\
& \underline{\mathrm{u}}_{\mathrm{e}}+{ }^{14} \mathrm{~N} \rightarrow{ }^{14} \mathrm{C}+\mathrm{e}^{+}, \mathrm{Q}=-1.18 \mathrm{MeV} .
\end{aligned}
$$

Because the $Q$-value for the second reaction is so much less negative than it is for the first, and the $U_{e}$ and $\underline{u}_{e}$ energies are of the same order as those Q-values, the second reaction is much more 
probable than the first. Furthermore, as noted above, the mean energy of the $\mathrm{U}_{\mathrm{e}} \mathrm{s}$ is expected to be less than that of the $\underline{u}_{\mathrm{e}} \mathrm{S}$, thereby enhancing the effect.

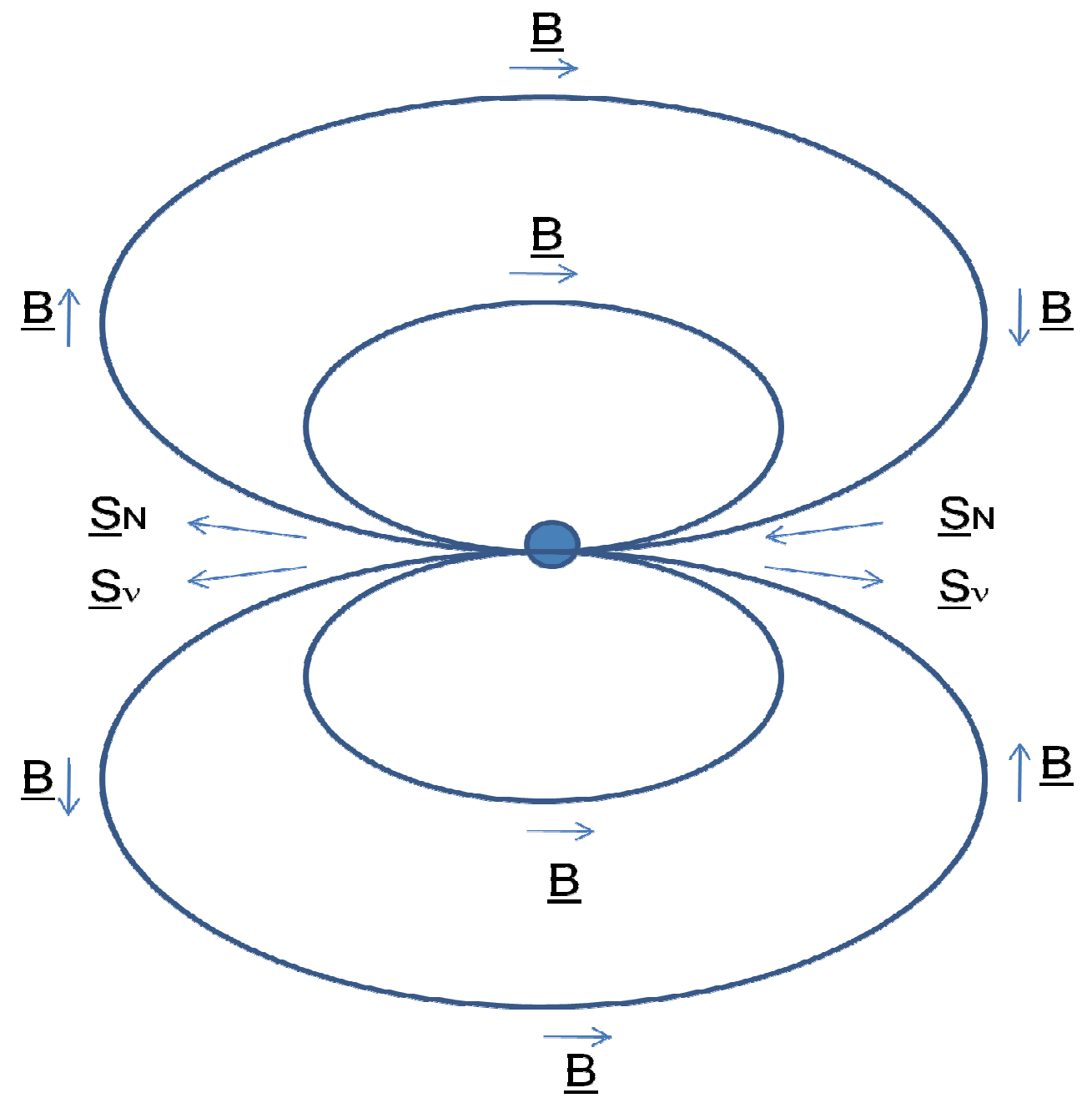

Figure 1. The magnetic field lines, $B$, and the spin directions of the electron antineutrino, $\underline{S}_{U}$, and ${ }^{14} N, \underline{S}_{N}$, in the region surrounding a neutron star or black hole (small sphere in the center).

However, the orientation of the spins is important. In both reactions, the final nucleus has a spin of zero, so, since the spin of the electron or positron is $\hbar / 2$, the total angular momentum will also be $\hbar / 2$. Thus the reactions can go without additional angular momentum if the total spin inthe entrance channel is also $\hbar / 2$, but will require an additional $1 \hbar$ in the case when the total spin is $3 \hbar / 2$. This must come from the wave functions of the electron/positron or from the neutrino/antineutrino, which is known from nuclear $\beta$-decay to result in an inhibition of roughly an order of magnitude (14). Assuming that the ${ }^{14} \mathrm{~N}$ spin is coupled to the chirality of the amino acid, this will produce a net selection of the molecular chirality that is not destroyed on one side of the neutron star or black hole, and no selection on the other, resulting in a net overall chiral selection.

The explanation for the coupling of the ${ }^{14} \mathrm{~N}$ spin to the molecular chirality was given $(16,17)$ in the context of nuclear magnetic resonance. The effect of the external magnetic field is to induce a 
current density which, in chiral samples, causes a nuclear magnetic moment, that of the ${ }^{14} \mathrm{~N}$, to induce an electric dipole moment in the molecule in which the ${ }^{14} \mathrm{~N}$ resides. The result is an effect that is opposite in sign for left-handed and right-handed molecules. A critical component of this argument is the non-zero-spin ${ }^{14} \mathrm{~N}$ nucleus.

\section{Amplifying Enantiomerism}

As shown below, the level of enantiomerism that could be produced in the SNAAP model is tiny, but it is at least as large as that from other models. Thus, all models proposed for producing net amino acid chirality requires some means for amplification of that enantiomerism, probably both in the cosmos and again once the molecules begin their planetary existence. Fortunately, these mechanisms appear to exist (18), and have even been demonstrated in the laboratory in some instances. One can estimate the fraction of a galaxy that could be processed by its supernovae by assuming a supernova rate, assuming that the processing volume of any single supernova will end at the point at which the magnetic field from that supernova is equal to the ambient galactic field, and then comparing that volume to the galactic volume. That ratio is extremely small (12), thereby necessitating amplification.

A basic component of amplification is "auto-catalysis" (3), by which chiral molecules replicate themselves in new molecules having the same chiral state. It is thought that the complex molecules that formed in space, and were subsequently observed by astronomers (19), may form on the icy surfaces of dust grains $(20,21)$, on which the amplification would presumably also occur. However, given the extremely small galactic volume that could be processed by supernovae, some mechanism for spreading the enantiomeric material throughout at least some of the galaxy also is required, if one chirality is to prevail. These mechanisms have been discussed (22); they include wind-blown bubbles, starburst superwinds, and several other mechanisms that have been identified by astronomers.

A number of experiments have been conducted to demonstrate that amplification of preexisting enatiomerism can occur in a variety of chiral molecules in environments that require water and some basic chemicals (see a discussion of these in ref. 12). The pre-existing enantiomeric excess, ee $=(\mathrm{L}-$ $R) /(L+R) \times 100$, in these experiments was as small as a fraction of a percent, but it is not clear that is the minimum required value; it could be much smaller. The crucial conclusion is that mechanisms for amplification both in outer space and in a planetary environment do exist, and appear to be capable of producing the enantiomerism of $100 \%$ that exists.

\section{Molecular Survival}

An important consideration, though, in the SNAAP model is the survival of the molecules, both from the photons from the star that is the progenitor of the supernova, and the photons from the supernova after it has exploded. Basic considerations of the maximum volume that can be processed by the supernova neutrinos in the supernova magnetic field suggest that supernovae that are produced by progenitor stars with masses less than about 25 solar masses could not possibly allow chemical survival, due to both the progenitor star and the supernova. The progenitor would become a red giant prior to its becoming a supernova, which would envelope the maximum processing distance, and the photon flux, though much less intense than the neutrino flux, has a much larger cross section for interaction with the molecules. However, more massive stars have "Wolf-Rayet" progenitors (23), which are much smaller (and much hotter!) stars, but which expel their outer layers to shield the region around them from their 
very hot stars at the center. While it would not be expected that molecules that existed for a long time in a Wolf-Rayet cloud would survive, molecules that were passing through, and which were contained in meteoroids, could survive. Furthermore, when such stars become supernovae, they produce black holes (24), instead of neutron stars, and the black holes would swallow much of the radiation that was produced by the explosion. Thus it appears that Wolf-Rayet progenitors combined with black hole final states would provide the environment in which the neutrino processing could occur without destroying the molecules, especially on amino acids that were contained in meteoroids that were passing through the Wolf-Rayet cloud when the supernova occurred.

\section{Probability of Producing Chiral Molecules}

The probability of processing a molecule via the neutrino induced interactions described above can be estimated using an estimated cross section for converting ${ }^{14} \mathrm{~N}$ to ${ }^{14} \mathrm{C}(25)$, and assuming that the separation from the core of the star as it becomes a supernova is $10^{11} \mathrm{~cm}(12)$. The assumption here is that the molecules would be contained within a large enough meteoroid that some of it could be ablated away during the time it passed by the Wolf-Rayet star without destroying all the molecules within it. The enantiomeric excess, ee, is then found to be $0.5 \times 10^{-6}$, although this could be somewhat larger due to the fact that the energies of the $\underline{u}_{\mathrm{e}} \mathrm{s}$ may be higher than used in this estimate (26) and, as shown by numerical simulations, there may be more $\underline{u}_{\mathrm{e}} \mathrm{s}$ than assumed from the fallback onto the newly formed neutron star or before the appearance of the black hole horizon $(27,28)$. This maximum ee is the quantity of interest, since subsequent auto-catalysis will presumably begin in the regions in which the ee was the largest.

\section{Producing the Other Chirality}

In the above discussion we ignored the possibility that $\mathrm{U}_{\mathrm{e}} \mathrm{s}$ would produce ${ }^{14} \mathrm{O}$, although they would certainly produce an excess thereof at the opposite end of the neutron star/black hole from that at which the $\underline{u}_{\mathrm{e}} \mathrm{S}$ selectively converted ${ }^{14} \mathrm{~N}$ to ${ }^{14} \mathrm{C}$. They would not be as efficient in converting ${ }^{14} \mathrm{~N}$ to ${ }^{14} \mathrm{O}$ as the $\underline{u}_{\mathrm{e}} \mathrm{S}$ were in converting ${ }^{14} \mathrm{~N}$ to ${ }^{14} \mathrm{C}$, so there would be a net chirality selection. However, the $\mathrm{U}_{\mathrm{e}} \mathrm{S}$ would produce regions of space in which the opposite chirality did dominate. Thus it might be expected that some meteoroids might contain inclusions in which right-handed amino acids would dominate, despite the fact that the regions in which the left-handed amino acids dominated would be more numerous. However, this is an important prediction of the model; a large sample of meteoroids should produce both chiralities if the SNAAP model is correct. The circularly polarized light models, by contrast, would predict some regions dominated by left-handed molecules, and an equal number of regions dominated by right-handed molecules.

We may have an answer soon as to which model is preferred. Space probe Hayabusa (29) should contain some material from comet Itokawa, although it will be gaseous, so would be mixed. Space probe ROSETTA (30) should send its lander to comet 67P/Churyumov-Gerasimenko in 2014, and should then produce analyses of solid samples in that comet. This may provide a definitive answer to the selection questions posed by the two models discussed above.

An interesting question exists as to whether or not the amino acid chirality that exists on Earth would be the same as that on all other planets. Certainly the SNAAP model suggests that the entire galaxy would be skewed toward a single chirality, but at a very small level of enantiomerism, and that 
regions of space surrounding a supernova would have material of the other chirality. It is thought that, early in the history of our Earth, an enormous bombardment of meteorites occurred (31); if that number was sufficiently large, even if the fraction of the meteorites that contained left-handed amino acids was small, there would be a small preference which would presumably become the only chirality following amplification. So the preference of left-handedness on other planets might depend on the intensity of the meteorite showers that occurred on them.

VIII. Summary

The SNAPP model is capable of producing global enantiomerism, although it would produce different regions of space in which each amino acid chirality would dominate. However, there would be one chirality that would be preferred overall. The steps by which the SNAAP model operates is illustrated in figure 2 , finally producing $100 \%$ chiral amino acids.

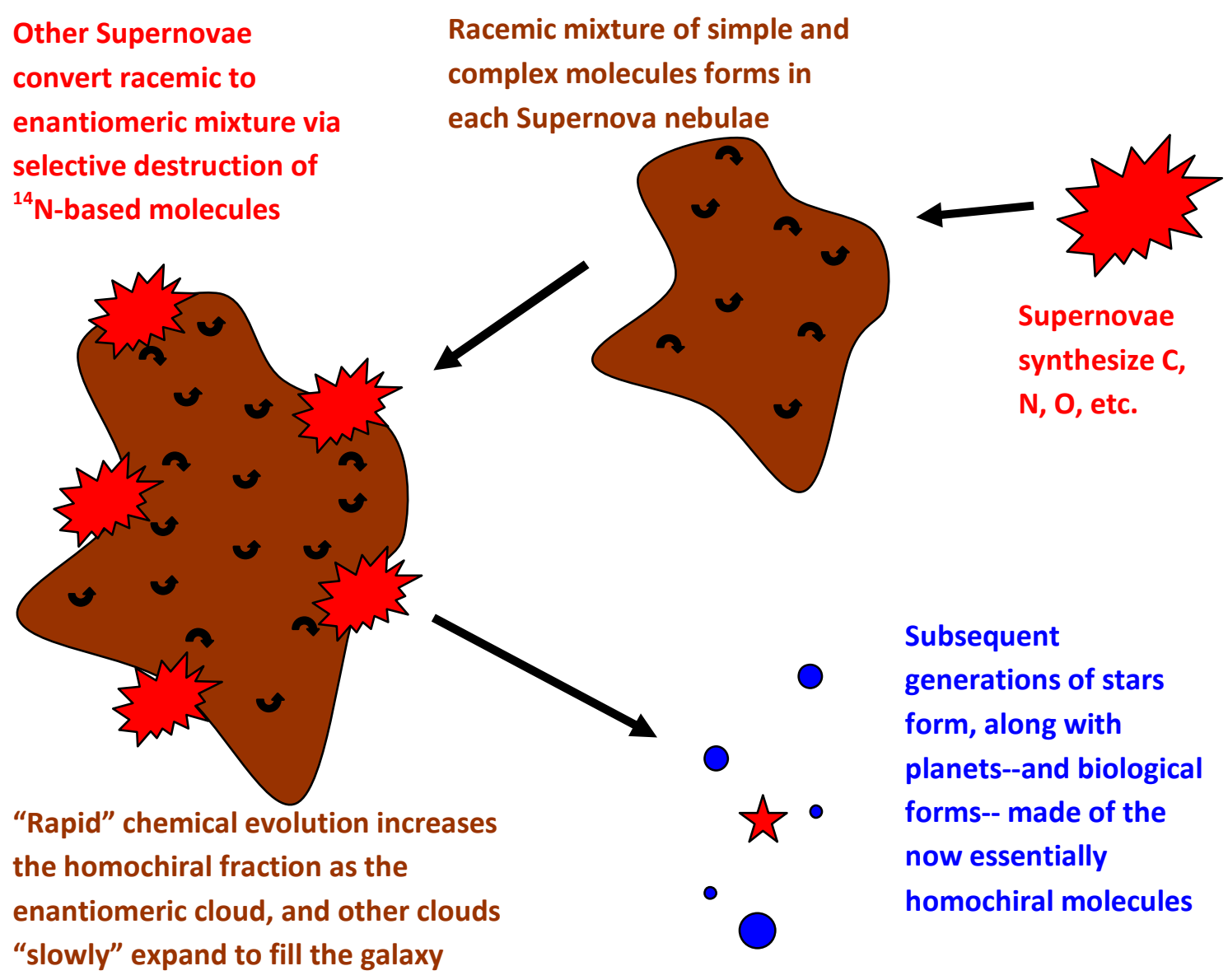

Figure 2. The components of the SNAAP model, beginning with production of the elements in supernovae, then formation of molecules, then chiral selection by more supernovae, then amplification of the chirality that was selected, and finally, with formation of planets and living beings.

Acknowledgements

This work was performed partially under the auspices of the U.S. Department of Energy by Lawrence Livermore National Laboratory under Contract DE-AC52-07NA27344, and partially with the support of 
Grants-in-Aid for Scientific Research (20244035) and on Innovative Areas (20105004) of the Ministry of Education, Culture, Sports, Science and Technology of Japan, and also by JSPS Core-to-Core Program, International Research Network for Exotic Femto Systems (EFES).

\section{References}

1. Miller, S.L., Science 117, 528 (1953); Miller, S.L. and Urey, H.C., Science 130, 245 (1959)

2. Bonner, W., Origins of Life and Evolution of Biospheres 21, 59 (1991)

3. Goldanskii, V.I. and Kuzmin, V.V., Sov. Phys. Usp. 32, 1 (1989)

4. Cronin, J.R., Pizzarello, S., \& Cruikshank, D.P., Meteorites and the Early Solar System, eds J.F. Kerridge and M.S. Matthews, University of Arizona Press, (Tucson, 1988) p. 819

5. Cronin, J.R. and Pizzarello, S., Science Magazine 275, 951 (1997)

6. Chyba, C.F., Nature 348, 113 (1990)

7. Bailey, J., Chrysostomou, A., Hough, J.H., Gledhill, T.M., McCall, A., Clark, S., Ménard, F., \& Tamura, M., Science 281, 672 (1998)

8. Takano, Y. et al., Earth Planet. Sci. Lett., 254, 106-114 (2007)

9. Bailey, J., Origins Life and Evolution of Biospheres 31, 0169 (2001)

10. Vester, F, Ulbright, T.L.V., and Krauch, H., Naturwissenschaften 46, 68 (1959)

11. Mann, A.K. \& Primakoff, H., Origins of Life 11, 255 (1981)

12. Boyd, R.N., Kajino, T., and Onaka, T., Astrobiology, to be published, 2010; ibid, arXiv:1001.3849

13. Arnett, D. Supernovae and Nucleosynthesis, Princeton U. Press, Princeton, 1996

14. Boyd, R.N., An Introduction to Nuclear Astrophysics, U. Chicago Press, Chicago, 2008

15. Yoshida, T., Kajino, T., and Hartmann, D.H., Phys. Rev. Lett. 94, 231101 (2005)

16. Buckingham, A.D., Chem. Phys. Letters 398, 1 (2004)

17. Buckingham, A.D. and Fischer, P., Chem.. Phys. 324, 111 (2006)

18. Glavin, D.P. and Dworkin, J.P., Proc. Nat. Acad. Sciences Early Edition (2009) www.pnas.org/cgi/doi/10.1073/pnas.0811618106

19. http://en.wikipedia.org/wiki/List of molecules in interstellar space

20. Hasegawa, T.I., Herbst, E., \& Leung, C.M., Astrophys. J. Suppl. Series 82, 167 (1992)

21. Garrod. R.T., Weaver, S.L.W., \& Herbst, E., Astrophys. J. 682, 283 (2008)

22. Pittard, J.M., Diffuse Matter from Star Forming Regions to Active Galaxies-A Volume Honouring John Dyson, ed. by T.W. Hartquist, J.M. Pittard, and S.A.E.G. Falle, Springer Dordrecht (2007)

23. Crowther, P.A., Ann. Rev. Astron. Astrophys. 45, 177 (2007)

24. Heger, A. et al., Astrophys. J. 591, 288 (2003)

25. Fuller, G.M., Haxton, W.C., and McLaughlin, G.C, Phys. Rev. D 59, 085005 (1999)

26. Lunardini, C., Phys. Rev. Letters 102, 231101 (2009)

27. Fryer, C.L., Astrophys. J. 699, 4009 (2009)

28. Sumiyoshi, K., et al., Phys. Rev. Lett. 97, 091101 (2006)

29. Yano, H. et al., Science 312, 1350 (2006)

30. Thiemann, W.H.-P., and Meierhenrich, U., Origins of Life and Evolution of Biospheres 31, 199 (2001)

31. Ehrenfreund, P., et al., Rep. Prog. Phys. 65, 1427 (2002) 